Network Working Group

Request for Comments: 3523

Category: Informational

\title{
Internet Emergency Preparedness (IEPREP) \\ Telephony Topology Terminology
}

Status of this Memo

This memo provides information for the Internet community. It does not specify an Internet standard of any kind. Distribution of this memo is unlimited.

Copyright Notice

Copyright (C) The Internet Society (2003). All Rights Reserved.

Abstract

This document defines the topology naming conventions that are to be used in reference to Internet Emergency Preparedness (IEPREP) phone calls. These naming conventions should be used to focus the IEPREP Working Group during discussions and when writing requirements, gap analysis and other solutions documents.

Table of Contents

1. Introduction. . . . . . . . . . . . . . . . . . . . . . . 2

1.1 Motivation. . . . . . . . . . . . . . . . . . . . 2

1.2 Terms and Definitions . . . . . . . . . . . . . . . . 2

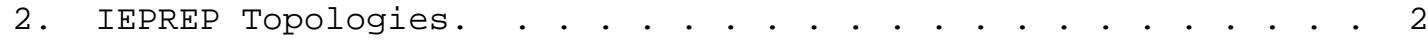

2.1 Topology "IP Bridging" . . . . . . . . . . . . . . . 3

2.2 Topology "IP at the Start". . . . . . . . . . . . . . 3

2.3 Topology "IP at the End". . . . . . . . . . . . . . . . 4

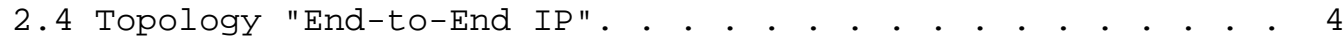

3. Security Considerations . . . . . . . . . . . . . . . 5

4. IANA Considerations . . . . . . . . . . . . . . . . . . . 5

5. Acknowledgements . . . . . . . . . . . . . . . . . . 5

6. References . . . . . . . . . . . . . . . . . . . . . . . 5

7. Author's Address. . . . . . . . . . . . . . . . . . . 5

8. Full Copyright Statement. . . . . . . . . . . . . 6 


\section{Introduction}

This document defines the topology naming conventions that are to be used in reference to IEPREP phone calls. These naming conventions should be used to focus the IEPREP Working Group (WG) during discussions and when writing requirements, gap analysis and other solutions documents.

There has been much confusion on the IEPREP list as well as within each meeting about the topologies IEPREP is considering. Hopefully this document will give each reader and author a reference set of named architectures.

This memo attempts to be agnostic with regard to IP signaling or control protocols (SIP, MEGACO, etc), as well as any underlying Quality of Service (QOS) mechanisms (Diffserv, RSVP, NSIS, etc).

1.1 Motivation

Simply put, to get everyone referencing the same (named) topologies in order to have useful and less confusing dialog to further this working group's efforts.

1.2 Terms and Definitions

The following acronyms need to be exploded for clarity:

$$
\begin{aligned}
& \text { CSN }=\text { Circuit Switched Network } \\
& \text { GW = Gateway (CSN to IP, or IP to CSN) }
\end{aligned}
$$

2. IEPREP Topologies

There are 4 often mentioned, but very little documented topologies discussed within this W's efforts so far. The following subsections name and describe each of the topologies.

The 4 topologies are (quickly):

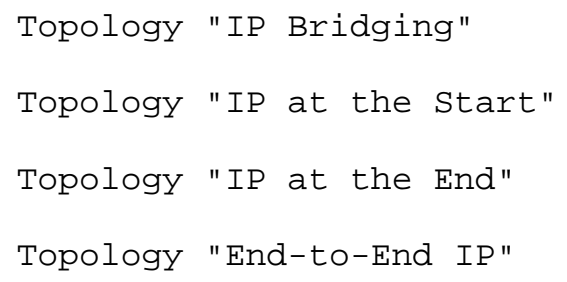




\subsection{Topology "IP Bridging"}

This topology is sometimes known as "IP in the Middle" of two CSNs. In this topology, a CSN phone of any type initiates (dials) a call to another CSN phone with an IP core between the two CSNs.

This topology should simplistically look like this:

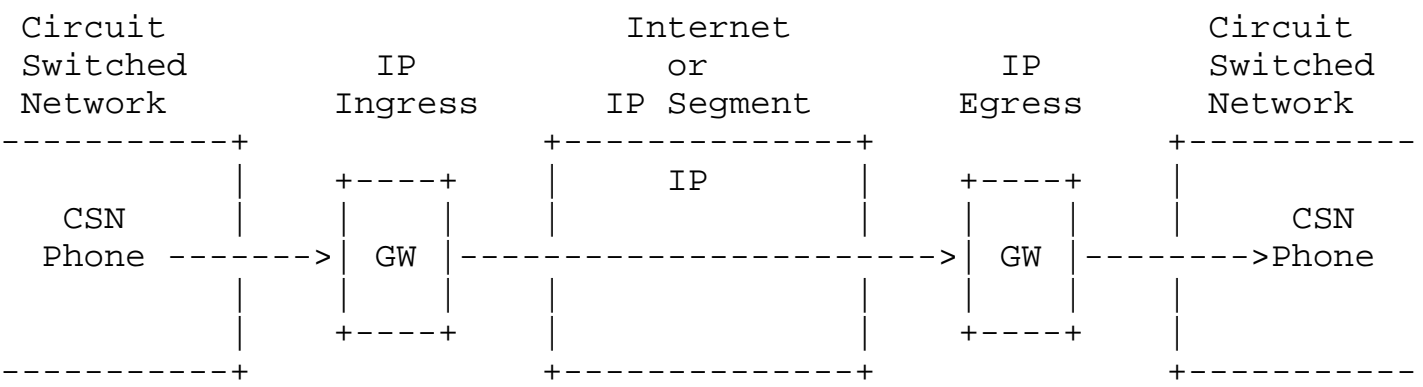

Figure 1. Topology "IP Bridging"

2.2 Topology "IP at the start"

This topology has the initiating party placing (dialing) the call

from an IP Phone (PDA or computer), and the called party residing in the CSN.

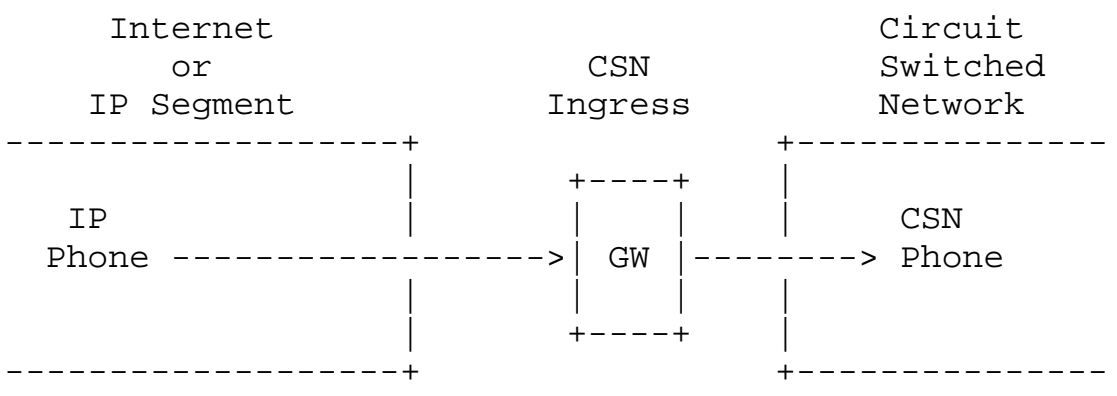

Figure 2. Topology "IP at the start" 


\subsection{Topology "IP at the End"}

This topology has the calling party placing the call from a CSN phone, and the called party being in an IP network.

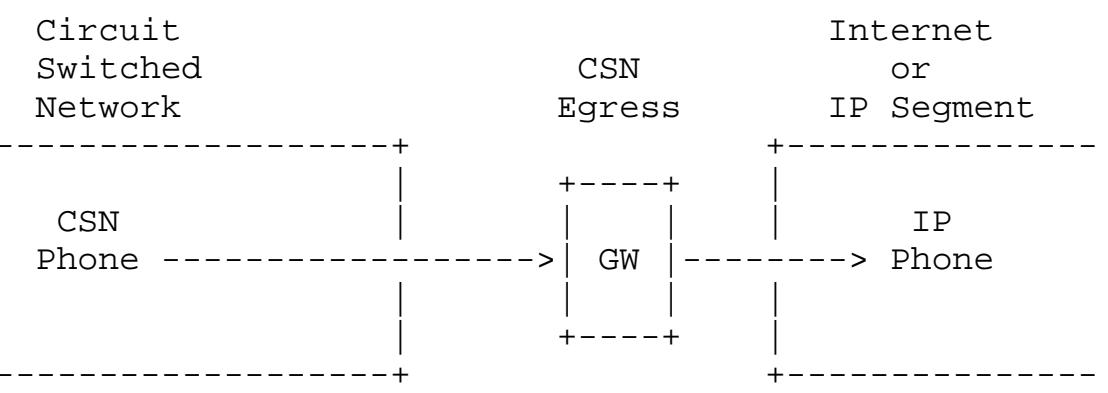

Figure 3. Topology "IP at the End"

2.4 Topology "End-to-End IP"

This topology has no circuit switched sections in the call path.

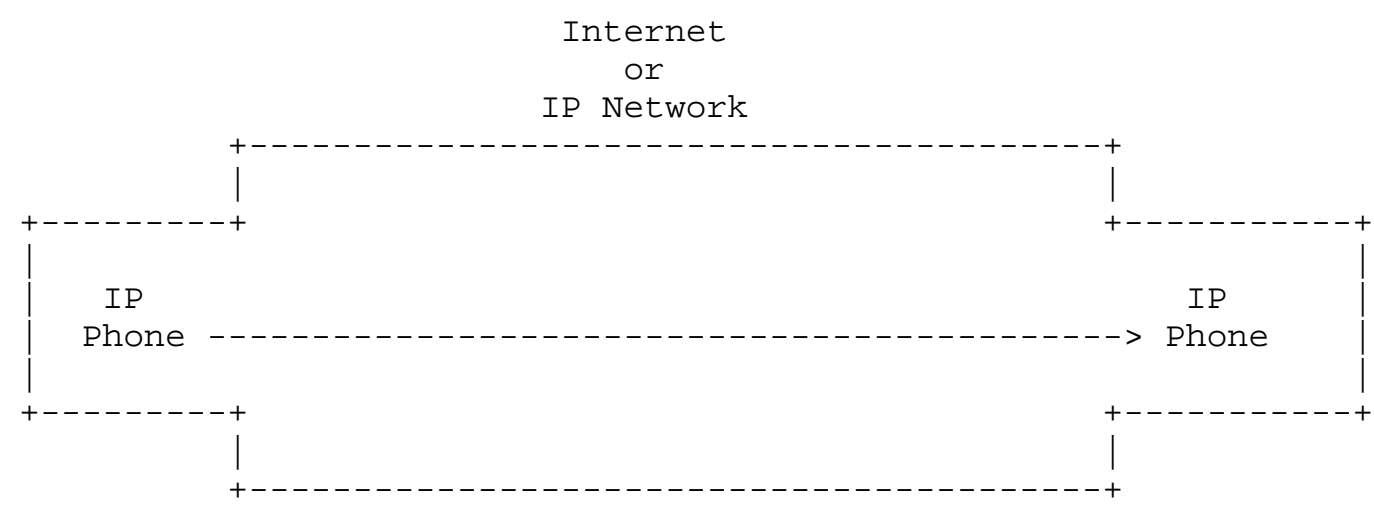

Figure 4. Topology "End to End IP"

Although shown as one large IP cloud here, the Internet is composed of a series of loosely connected IP domains. An End-to-End IP call will likely traverse a number of these domains and/or multiple network providers, which may impact the call. 
3. Security Considerations

This document merely suggests a common naming convention within IEPREP WG discussions, therefore there are no special security considerations.

4. IANA Considerations

There are no IANA considerations within this document.

5. Acknowledgements

To Scott Bradner, Kimberly King and Mike Pierce for their comments and suggestions.

6. References

There are no references at the present time.

7. Author's Address

James M. Polk

Cisco Systems

2200 East President George Bush Turnpike

Richardson, Texas 75082 USA

EMail: jmpolk@cisco.com 
8. Full Copyright statement

Copyright (C) The Internet Society (2003). All Rights Reserved.

This document and translations of it may be copied and furnished to others, and derivative works that comment on or otherwise explain it or assist in its implementation may be prepared, copied, published and distributed, in whole or in part, without restriction of any kind, provided that the above copyright notice and this paragraph are included on all such copies and derivative works. However, this document itself may not be modified in any way, such as by removing the copyright notice or references to the Internet society or other Internet organizations, except as needed for the purpose of developing Internet standards in which case the procedures for copyrights defined in the Internet Standards process must be followed, or as required to translate it into languages other than English.

The limited permissions granted above are perpetual and will not be revoked by the Internet society or its successors or assigns.

This document and the information contained herein is provided on an "AS IS" basis and THE INTERNET SOCIETY AND THE INTERNET ENGINEERING TASK FORCE DISCLAIMS ALL WARRANTIES, EXPRESS OR IMPLIED, INCLUDING BUT NOT LIMITED TO ANY WARRANTY THAT THE USE OF THE INFORMATION HEREIN WILL NOT INFRINGE ANY RIGHTS OR ANY IMPLIED WARRANTIES OF MERCHANTABILITY OR FITNESS FOR A PARTICULAR PURPOSE.

Acknowledgement

Funding for the RFC Editor function is currently provided by the Internet society. 